PROCEEDINGS OF THE

AMERICAN MATHEMATICAL SOCIETY

Volume 128, Number 5, Pages 1313-1317

S 0002-9939(00)05624-0

Article electronically published on February 7, 2000

\title{
DECIDABILITY OF THE REPRESENTATION EXTENSION PROPERTY FOR FINITE SEMIGROUPS
}

\author{
KUNITAKA SHOJI
}

(Communicated by Ronald M. Solomon)

\begin{abstract}
We prove that the decision problem of whether or not a finite semigroup has the representation extension property is decidable.
\end{abstract}

\section{THE MAIN THEOREM AND PRELIMINARIES}

It is an immediate consequence of the normal form theorem for amalgamated free products of groups that every amalgam of groups embeds in some group. However, this result fails for semigroup amalgams: an early result of Kimura [6] shows that amalgams of semigroups do not necessarily embed in any semigroup (see also [4, Vol. II, page 138). More recently, Sapir [7] has shown that it is in fact undecidable whether an amalgam of (finite) semigroups embeds in any (finite) semigroup. A semigroup $S$ is called an amalgamation base for semigroups if every amalgam of semigroups containing $S$ as a subsemigroup embeds in some semigroup. It is natural to ask if it is decidable whether or not a finite semigroup is an amalgamation base.

According to [3], we say that a semigroup $S$ has the representation extension property if for any right $S$-set $X_{S}$ and any left $S$-set ${ }_{S} M$ containing $S^{1}$ as a left $S$ subset, the canonical map: $X \rightarrow X \otimes_{S} M(x \longmapsto x \otimes 1)$ is injective. Hall [5] proved that any semigroup which is an amalgamation base in the class of all semigroups has the representation extension property. In this paper we prove

The Main Theorem. It is decidable whether or not a finite semigroup has the representation extension property.

Let $S$ be a semigroup. Let $M$ be a nonempty set with a unitary and associative operation of $S: S^{1} \times M \longrightarrow M((s, w) \longmapsto s w)$, where $S^{1}$ is the monoid obtained from $S$ by adjoining a new identity 1 . Then $M$ is called a left $S$-set. Dually, a right $S$-set is defined. If a left $S$-set [resp. right $S$-set] $M$ contains elements $m_{1}, \cdots, m_{n}$ such that $M=S^{1} m_{1} \cup \cdots \cup S^{1} m_{n}$ [resp. $M=m_{1} S^{1} \cup \cdots \cup m_{n} S^{1}$ ], then we say that $m_{1}, \cdots, m_{n}$ are generators of $M$.

A relation $\rho$ on a left [resp. right] $S$-set $M$ is called an $S$-congruence if $\left(m, m^{\prime}\right)$ $\in \rho$ and $s \in S$ implies $\left(s m, s m^{\prime}\right) \in \rho$ [resp. $\left(m s, m^{\prime} s\right) \in \rho$ ]. Let $M, N$ be left [resp.

Received by the editors July 1, 1998.

1991 Mathematics Subject Classification. Primary 20M10.

Key words and phrases. Finite semigroup, representation extension property, algorithm, decidability.

This work was done while the author was visiting the University of Nebraska, Lincoln. He thanks the Department of Mathematics, UNL for their kind hospitality. 
right] $S$-sets. Then a map $\phi: M \longrightarrow N$ is called an $S$-map if $\phi(s m)=s \phi(m)$ [resp. $\phi(m s)=\phi(m) s]$ for any $m \in M$ and $s \in S$.

Result (4, Proposition 1.5]). Let $S$ be a semigroup and $A, B, C, D$ left [resp. right] $S$-sets such that $C$ is a left [resp. right] $S$-subset of $A$ and $D$ is a left [resp. right] $S$-subset of $B$. Let $\alpha$ be a bijective $S$-map: $C \longrightarrow D$. Then there exist a left [resp. right] $S$-set $W$ and injective $S$-maps $\beta: A \longrightarrow W, \lambda: B \longrightarrow W$ such that $\alpha \lambda=\beta$ on $C, W=A \beta \cup B \lambda, A \beta \cap B \lambda=C \beta$.

In this case, we say that the left [resp. right] $S$-set $W$ is the left [resp. right] $S$-set obtained by gluing $A$ and $B$ with $\alpha$ and write $W=A \#_{\alpha} B$.

If $C, D$ are generated by $x$ and $y$ respectively and $\alpha(x)=y$, then we write $A$ $\#_{x=y} B$ instead of $A \#_{\alpha} B$.

Let $X$ be a right $S$-set. Consider a set $\Sigma$ of equations $x_{i} t_{i}=x_{i+1} s_{i+1}(1 \leq i \leq$ $n-1)$ on $X$, where $t_{i}, s_{i+1} \in S, x_{i} \in X$. Then for any $1 \leq i \leq n$, we define the $S$-congruence $\rho_{i}$ on the right $S$-set $S^{1}$ as follows:

$(s, t) \in \rho_{i}$ if and only if $x_{i} s=x_{i} t$ in $X$ for all $s, t \in S^{1}$.

Let $\overline{X_{i}}=S^{1} / \rho_{i}$ and $\overline{x_{i}}=1 \rho_{i}$. Then we can obtain a right $S$-set $\bar{X}$ such that

$$
\bar{X}=\bar{X}_{1} \# \bar{x}_{1} t_{1}=\bar{x}_{2} s_{2} \bar{X}_{2} \cdots \# \bar{X}_{i-1} \# \bar{x}_{i t_{i}=\bar{x}_{i+1} s_{i+1}} \bar{X}_{i+1} \# \cdots \# \bar{x}_{\bar{x}_{n-1} t_{n-1}=\bar{x}_{n} s_{n}} \bar{X}_{n} .
$$

Then we have the set of equations $\bar{x}_{i} t_{i}=\bar{x}_{i+1} s_{i+1}(1 \leq i \leq n-1)$ in $\bar{X}$. We call $\bar{X}$ the relatively free right $S$-set associated to $X$ with respect to $\Sigma$.

\section{DECISION PROBLEM FOR THE REPRESENTATION EXTENSION PROPERTY}

Let $S$ be a finite semigroup and let $\mathcal{T}\left(S^{1}\right)$ denote the set of all mappings of $S^{1}$. Then we define an operation of $S$ on $\mathcal{T}\left(S^{1}\right): t f$ is defined by $(s) t f=(s t) f$ for all $s, t \in S^{1}$ and $f \in \mathcal{T}\left(S^{1}\right)$. Then $\mathcal{T}\left(S^{1}\right)$ is a left $S$-set.

Next let $\rho: S^{1} \rightarrow \mathcal{T}\left(S^{1}\right)$ denote the right regular representation of $S^{1}$. Then by $\rho$ we regard the left $S$-set $S^{1}$ is a left $S$-subset of the left $S$-set $\mathcal{T}\left(S^{1}\right)$. By [1. Theorem 6], the left $S$-set $\mathcal{T}\left(S^{1}\right)$ is injective. As a consequence, we have (see Remark after Corollary 2.3 of [8])

Lemma. Let $S$ be a finite semigroup and $\mathcal{T}\left(S^{1}\right)$ as above. Then $S$ has the representation extension property if and only if for any right $S$-set $X_{S}$, the canonical map: $X \rightarrow X \otimes_{S} \mathcal{T}\left(S^{1}\right)(x \longmapsto x \otimes 1)$ is injective.

Proof. This follows from Theorem 2.1 of $[8]$ by substituting $\mathcal{T}\left(S^{1}\right)$ for the left $S$-set $W$.

Then we shall prove that for any finite semigroup $S$, there is a positive integer $k$ dependinig only on the order $|S|$ of $S$ such that if there exist a right $S$-set $X$ and a scheme of length greater than $k$ which shows $x \otimes 1=x^{\prime} \otimes 1$ in $X \otimes_{S} \mathcal{T}\left(S^{1}\right)$ but $x \neq x^{\prime}$ in $X$, then there exist a right $S$-set $Y$ with fewer generators than $k$ and $y, y^{\prime} \in Y$ such that $y \otimes 1=y^{\prime} \otimes 1$ in $Y \otimes_{S} \mathcal{T}\left(S^{1}\right)$ but $y \neq y^{\prime}$ in $Y$ (see [2] for undefined terms).

Proof of the main theorem. Let $S$ be a finite semigroup. By the lemma, suppose that there exist a right $S$-set $X$ and $x, x^{\prime} \in X$ such that $x \neq x^{\prime}$ in $X$ and $x \otimes 1$ $=x^{\prime} \otimes 1$ in $X \otimes_{S} \mathcal{T}\left(S^{1}\right)$. Then by Lemma 1.2 of [2], there exist $x_{1}, \cdots, x_{n} \in X$, 
$y_{2}, \cdots, y_{n} \in \mathcal{T}\left(S^{1}\right), s_{1}, \cdots, s_{n}, t_{1}, \ldots, t_{n} \in S$ such that

\begin{tabular}{|c|c|c|c|c|c|}
\hline$x$ & $=$ & $x_{1} s_{1}$ & $s_{1}$ & $=$ & $t_{1} y_{2}$ \\
\hline$x_{1} t_{1}$ & $=$ & $x_{2} s_{2}$ & $s_{2} y_{2}$ & $=$ & $t_{2} y_{3}$ \\
\hline$x_{n-1} t_{n-1}$ & $=$ & $x_{n} s_{n}$ & $s_{n} y_{n}$ & $=$ & $t_{n}$ \\
\hline$x_{n} t_{n}$ & $=$ & $x^{\prime}$. & & & \\
\hline
\end{tabular}

Then we can assume that $X=\bar{X}$, the relatively free right $S$-set of the right $S$-set associated to $X$ with respect to the set of equations (1). Thus we have

$$
\begin{gathered}
X=X_{1} \# x_{1} t_{1}=x_{2} s_{2} \\
X_{2} \# \cdots \# X_{i} \#_{x_{i} t_{i}=x_{i+1} s_{i+1}} X_{i+1} \\
\# \cdots \# X_{n-1} \# x_{n-1} t_{n-1}=x_{n} s_{n} X_{n} .
\end{gathered}
$$

Next we let $C_{r}(S)$ be the set of $S$-congruences on the right $S$-set $S^{1}$. In particular, let $\rho_{i}$ denote the $S$-congruence on the right $S$-set $S^{1}$ given by

$$
(s, t) \in \rho_{i} \text { if and only if } x_{i} s=x_{i} t \text { for all } s, t \in S .
$$

Let $m=\left|S \times C_{r}(S) \times S \times C_{r}(S) \times \mathcal{T}\left(S^{1}\right)\right|$ and $k=2 m$. If $n>k$, then there exist $1 \leq i_{1}<i_{2}<i_{3} \leq n$ such that

$$
\begin{aligned}
\left(t_{i_{1}}, \rho_{i_{1}}, s_{i_{1}+1}, \rho_{i_{1}+1}, y_{i_{1}+1}\right) & =\left(t_{i_{2}}, \rho_{i_{2}}, s_{i_{2}+1}, \rho_{i_{2}+1}, y_{i_{2}+1}\right) \\
& =\left(t_{i_{3}}, \rho_{i_{3}}, s_{i_{3}+1}, \rho_{i_{3}+1}, y_{i_{3}+1}\right),
\end{aligned}
$$

where $y_{n+1}=1$.

By the construction of $X$, we know that $X_{i}$ is $S$-isomorphic to $S^{1} / \rho_{i}$ as a right $S$-set with an $S$-map mapping $x_{i}$ on $1 \rho_{i}$. Then, for $(p, q)=(1,2),(1,3),(2,3)$, there are bijective $S$-maps:

$$
\begin{array}{r}
x_{i_{p}} t_{i_{p}} S \rightarrow x_{i_{q}} t_{i_{q}} S\left(x_{i_{p}} t_{i_{p}} \longmapsto x_{i_{q}} t_{i_{q}}\right), \\
x_{i_{p}+1} s_{i_{p}+1} S \longrightarrow x_{i_{q}+1} s_{i_{q}+1} S \quad\left(x_{i_{p}+1} s_{i_{p}+1} \longmapsto x_{i_{q}+1} s_{i_{q}+1}\right) \\
\text { and } \\
x_{i_{p}} t_{i_{p}} S \rightarrow x_{i_{q}+1} s_{i_{q}+1} S \quad\left(x_{i_{p}} t_{i_{p}} \longmapsto x_{i_{q}+1} s_{i_{q}+1}\right) .
\end{array}
$$

By $(5)$, for $(p, q)=(1,2),(1,3),(2,3)$, we obtain three right $S$-sets

$$
\begin{gathered}
X_{i_{p}, i_{q}}=X_{1} \# \bar{x}_{1} t_{1}=x_{2} s_{2} \bar{X}_{2} \# \cdots \# X_{i_{p-1}} \# x_{x_{i} t_{i_{p}}}=x_{i_{q}+1} s_{i_{q+1}} \\
\# \cdots X_{i_{q}+1} \\
\# \cdots x_{x_{n-1} t_{n-1}}=x_{n} s_{n} X_{n} .
\end{gathered}
$$

Then it follows from (1) and

$$
\left(t_{i_{p}}, \rho_{i_{p}}, s_{i_{p}+1}, \rho_{i_{p}+1}, y_{i_{p}+1}\right)=\left(t_{i_{q}}, \rho_{i_{q}}, s_{i_{q}+1}, \rho_{i_{q}+1}, y_{i_{q}+1}\right)
$$

that $x \otimes 1=x^{\prime} \otimes 1$ in $X_{i_{p}, i_{q}} \otimes_{S} \mathcal{T}\left(S^{1}\right)$ for all $1 \leq p<q \leq 3$. On the other hand, there must exist at least one pair $(p, q)$ such that $x \neq x^{\prime}$ in $X_{i_{p}, i_{q}}$. Suppose, on the contrary, that $x=x^{\prime}$ holds in $X_{i_{1}, i_{2}}, X_{i_{2}, i_{3}}$ and $X_{i_{1}, i_{3}}$. Then it follows from the first equation $x=x^{\prime}$ in $X_{i_{1}, i_{2}}$ and the way of construction of $X_{i_{1}, i_{1}}$ that

$$
x \in \bigcap_{i=1}^{i_{1}} x_{i} t_{i} S, \quad x^{\prime} \in \bigcap_{j=i_{2}+1}^{n} x_{j} s_{j} S
$$

and there exists $u \in S$ such that

$$
x=x_{i_{1}} t_{i_{1}} u \text { in } X_{1} \# x_{1} t_{1}=x_{2} s_{2} X_{2} \# \cdots \# X_{i_{1}}
$$


and

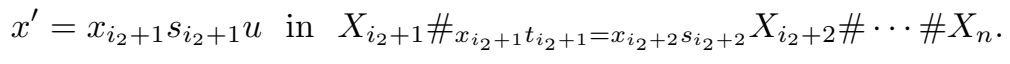

Similarly, it follows from the other equations in $X_{i_{2}, i_{2}}$ or $X_{i_{3}, i_{3}}$ that there exist $v, w \in S$ such that

$$
\begin{gathered}
x=x_{i_{2}} t_{i_{2}} v \text { in } X_{1} \# x_{x_{1} t_{1}=x_{2} s_{2}} X_{2} \# \cdots \# X_{i_{2}} \\
x^{\prime}=x_{i_{3}+1} s_{i_{3}+1} v \text { in } X_{i_{3}+1} \# x_{x_{3}+1} t_{i_{3}+1}=x_{i_{3}+2} s_{i_{3}+2} \\
X_{i_{3}+2} \# \cdots \# X_{n}
\end{gathered}
$$

and

$$
\begin{aligned}
& x=x_{i_{1}} t_{i_{1}} w \text { in } X_{1} \# x_{x_{1} t_{1}=x_{2} s_{2}} X_{2} \# \cdots \# X_{i_{1}},
\end{aligned}
$$

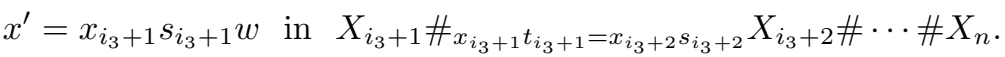

Of course, it can be assumed that

$$
X_{1} \# x_{x_{1} t_{1}=x_{2} s_{2}} X_{2} \# \cdots \# X_{i_{1}} \subseteq X_{1} \# x_{x_{1} t_{1}=x_{2} s_{2}} X_{2} \# \cdots \# X_{i_{2}}
$$

and

$$
\begin{aligned}
& X_{i_{3}+1} \# x_{i_{3}+1} t_{i_{3}+1}=x_{i_{3}+2} s_{i_{3}+2} X_{i_{3}+2} \# \cdots \# X_{n}
\end{aligned}
$$

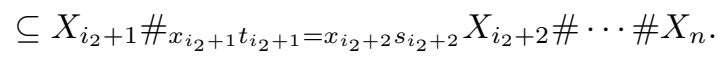

Consequently, we have

$$
x=x_{i_{1}} t_{i_{1}} u=x_{i_{2}} t_{i_{2}} v=x_{i_{1}} t_{i_{1}} w \text { in } X_{1} \# x_{x_{1} t_{1}=x_{2} s_{2}} X_{2} \# \cdots \# X_{i_{2}}
$$

and

$$
\begin{aligned}
& x^{\prime}=x_{i_{2}+1} s_{i_{2}+1} u=x_{i_{3}+1} s_{i_{3}+1} v=x_{i_{3}+1} s_{i_{3}+1} w \\
& \text { in } X_{i_{2}+1} \# x_{i_{2}+1} t_{i_{2}+1}=x_{i_{2}+2} s_{i_{2}+2} \\
& X_{i_{2}+2} \# \cdots \# X_{n} .
\end{aligned}
$$

Since $x_{i_{1}} t_{i_{1}} u=x_{i_{1}} t_{i_{1}} w$, by (3) we have $x_{i_{2}} t_{i_{2}} u=x_{i_{2}} t_{i_{2}} w$. Also, by (5) we have $x_{i_{2}+1} s_{i_{2}+1} u=x_{i_{2}+1} s_{i_{2}+1} w$. Since $x_{i_{3}+1} s_{i_{3}+1} v=x_{i_{3}+1} s_{i_{3}+1} w$, by (4) we have $x_{i_{2}+1} s_{i_{2}+1} v=x_{i_{2}+1} s_{i_{2}+1} w$. Therefore $x^{\prime}=x_{i_{2}+1} s_{i_{2}+1} u=x_{i_{2}+1} s_{i_{2}+1} v=$

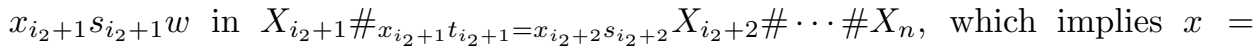
$x_{i_{2}} t_{i_{2}} u=x_{i_{2}} t_{i_{2}} v=x_{i_{2}} t_{i_{2}} w$ in $X_{1} \# x_{1} t_{1}=x_{2} s_{2} X_{2} \# \cdots \# X_{i_{2}}$, because of (4) and (5). Whence, by (2) $x=x_{i_{2}} t_{i_{2}} u=x_{i_{2}+1} s_{i_{2}+1} u=x^{\prime}$ in $X$. This is a contradiction. Consequently, in order to decide whether or not the lemma above holds for the finite semigroup $S$, it suffices to check whether or not the canonical map: $X \rightarrow X \otimes_{S} \mathcal{T}\left(S^{1}\right)$ is injective for all right $S$-sets $X$ with generators of its number equal to or less than $k$. Note that there exist only finitely many right $S$-sets with generators of its number equal to or less than $k$, up to $S$-isomorphism. Hence the main theorem follows.

As far as we know, the following problems remain open.

Problem. Is it decidable whether or not a finite semigroup has any one of the following properties?

(1) being an amalgamation base for semigroups.

(2) being an amalgamation base for finite semigroups.

\section{Acknowlegdments}

I thank John Meakin for his generous hospitality and stimulating conversation without which this work would not have been realized. I thank Tom Hall for reading the manuscript and correcting it. Finally, I thank the referee for good advice. 


\section{REFERENCES}

[1] P. Berthiaume, The injective envelope of S-sets, Canad. Math. Bull., 10(1967), 261-273. MR 35:4321

[2] S. Bulman-Fleming and K. McDowell, Absolutely flat semigroups, Pacific J. Math. 107(1983), 319-333. MR 85e:20056

[3] S. Bulman-Fleming and K. McDowell, Flatness and amalgamation in semigroups, Semigroup Forum 29(1984), 337-342. MR 85k:20198

[4] A. H. Clifford and G. B. Preston, Algebraic theory of semigroups, Amer. Math. Soc., Math. Survey, No.7, Providence, R.I., Vol.I(1961); Vol.II(1967). MR 24:A2627 MR 36:1558

[5] T. E. Hall, Representation extension and amalgamation for semigroups, Quart. J. Math. Oxford (2) 29(1978), 309-334. MR 80a:20084

[6] N. Kimura, On Semigroups, Ph.D thesis, Tulane University, 1957.

[7] M. V. Sapir, Algorithmic problems for amalgams of finite semigroups, preprint.

[8] K. Shoji, Amalgamation bases for semigroups, Math. Japonicae, 26(1990), 43-53. MR 91m:20090

Department of Mathematics, Shimane University, Matsue, Shimane, 690-8503 Japan

E-mail address: ksho@math.shimane-u.ac.jp 